\title{
Multinuclear MRI Research
}

\author{
Yu. A. Pirogov ${ }^{1}$ (D)
}

Received: 13 May 2021 / Revised: 19 July 2021 / Accepted: 20 July 2021 /

Published online: 31 July 2021

(c) The Author(s), under exclusive licence to Springer-Verlag GmbH Austria, part of Springer Nature 2021

\begin{abstract}
The presented data clearly demonstrate that multinuclear MRI has a great potential in research and clinical fields. The biomedical value of many heavy nuclei that are part of drugs, contrast agents, and molecular complexes in live tissues emphasizes the need for creating and using MRI scanners tuned to the Larmor frequencies of such nuclei. This article discusses a number of specific problems solved with the use of multinuclear MRI. Since the sensitivity of the MRI on heavy nuclei, as a rule, is insufficient for obtaining images of satisfactory quality, it is proposed to use hyperpolarization techniques for preparing objects for the MRI diagnostics. However, bearing in mind the high cost of hyperpolarization techniques, alternative approaches using contrast agents normally absent in tissues can be more suitable in certain situations. For instance, due to the absence of fluorine in the body, ${ }^{19} \mathrm{~F}$ MRI allows for successful diagnostics of the respiratory tract and lungs filled with fluorine-containing gas, detecting the location of the blood substitute Perftoran $₫$ in the body, monitoring the transport along the gastrointestinal tract of a capsule filled with a preparation containing fluorine-19 nuclei, etc. The possibility of non-invasive local measurements of the concentration of sodium-23 in the body, which, in case of a deviation from the norm, is linked to such pathologies as diabetes, hypertension, renal failure, and many others, is also demonstrated. Reported as well is the creation of transmitting and receiving infrastructure that makes possible effective MRI measurements at low fields (of about $0.5 \mathrm{~T}$ ) at 10 Larmor frequencies corresponding to the nuclei of hydrogen, deuterium, fluorine, boron, chlorine, sodium, carbon, phosphorus, silicon and oxygen.
\end{abstract}

Yu. A. Pirogov

yupi937@gmail.com

1 Faculty of Physics, M. V. Lomonosov Moscow State University, Moscow, Russia 


\section{Introduction}

Magnetic resonance imaging (MRI) is the most effective method of medical diagnostics due to the high information content and safety of the study. This is the only imaging method that, unlike PET, computed (X-ray), acoustic, or microwave tomography, does not require solving the inverse problem for obtaining the differential distributions of tissue density within the body. The use of direct algorithms makes MRI the most reliable way of biomedical diagnostics. Currently, popular optical and terahertz approaches are limited in imaging depth to only the very thin superficial layers (hundreds of micrometers thick) due to the strong attenuation of radiation within the tissue. Unlike them, MRI allows studying the characteristics of a live tissue at any depth within the body. But perhaps the most significant advantage of MRI over other noninvasive techniques is its ability, in combination with the local NMR spectroscopy, to study in real time the molecular structure of tissues and the behavior of drugs induced into the body in the course of therapeutic procedures. Although this approach is not fully mastered yet, it positions MRI as an advanced theranostics modality.

In clinical settings, the MRI signal is usually recorded only from hydrogen nuclei $\left({ }^{1} \mathrm{H}\right.$, protons). The high content of protons in soft tissue allows visualizing effectively its anatomical structure, which is hard to achieve with, e.g., computed tomography due to the transparency of soft tissue to X-ray radiation. However, some tissues have relatively low content of hydrogen atoms and produce weak MRI signal. For that reason, it is difficult to visualize airways, lungs, gastrointestinal tract, etc., using only the conventional proton MRI. Trying to visualize the location of administered drugs within the body is even more problematic, as some of them, like the blood substitute Perftoran ${ }^{\circledR}$, completely lack hydrogen atoms. Therefore, the task of developing MRI methods tuned to the frequencies of not only protons, but also heavier nuclei, so-called multinuclear magnetic resonance imaging, has become highly relevant. This article presents the results of the studies in the area of multinuclear MRI performed in the last decade by the interdisciplinary team of the Center for Magnetic Tomography and Spectroscopy (CMTS) of the Moscow State University (MSU). This Center was created by order of the Rector of the MSU, Academician Viktor Sadovnichy, exactly 25 years ago on March 19, 1996, as an independent interdepartmental and interdisciplinary division of the Moscow University.

With the support of the Rector, the Center was equipped with modern research equipment from the BRUKER company (Fig. 1). Due to the universal purpose of these devices, it began to act as a Center for Collective Use (CCU) and a complex of unique scientific instrumentation (USI), registered in the Ministry of Education and Science of the Russian Federation under the name "Biospectrotomography".

The equipment operates around the clock and is used for numerous research projects conducted not only by the MSU subdivisions but also by many Russian and international academic and industrial laboratories working jointly with the MSU scientists. Suffice to say that $3 \%$ of all publications by the MSU scientists in top-rated journals of the 1 st and 2 nd quartiles report the scientific results 

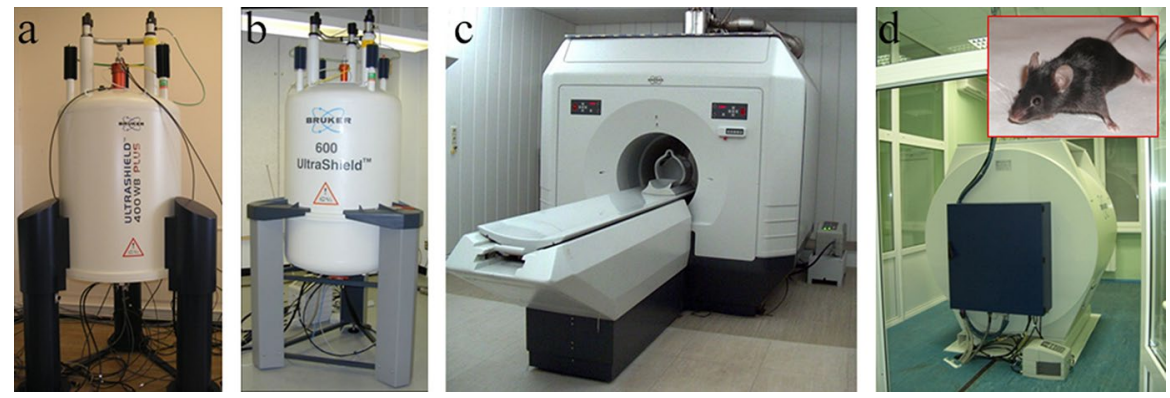

Fig. 1 The instrumentation of the CMTS MSU. a AV-400WB: 10-T solid-state NMR spectrometer at $400 \mathrm{MHz}$; b AV-600: 14.5-T $600 \mathrm{MHz}$ spectrometer; c Tomikon S50: 0.5-T MRI clinical scanner; d BioSpec 70/30 USR: 7-T biospectral scanner for the study of small laboratory animals

obtained on the equipment of the MSU CMTS. In 2010, the CMTS became a part of the Department of Fundamental Medicine of the MSU as an interdepartmental and interdisciplinary laboratory, keeping the same, well known by then, brand. Academician Aleksey Khokhlov is the head of this laboratory, as well as of the Center for Collective Use and the USI "Biospectrotomography" complex.

The staple tomographic device in the Center is a clinical low-field MRI scanner Tomikon S50 (Bruker, Karlsruhe, Germany), on which the main multinuclear studies were performed. Despite its relatively low magnetic field $(0.5 \mathrm{~T})$, this device is absolutely unique. Thanks to the permission of the BRUKER company, the research group of physicists led by Dr.Sci. Nikolay Anisimov got access to the software and infrastructure of this scanner and modified its for their research goals. The result was a whole series of new MRI methods dramatically improving the imaging not only at the Larmor frequency of protons $(21 \mathrm{MHz})$, but also at nine other resonance frequencies — of deuterium, fluorine, sodium, oxygen, boron, carbon, chlorine, silicon, and phosphorus. The created multinuclear infrastructure of the receiving and transmitting channel (Fig. 2) made this MRI scanner the only one in the world operating at 10 Larmor frequencies [1-7].

Presented here are some examples of the multinuclear techniques developed at the CMTS, starting with the proton one (Fig. 3), followed by others tailored for the detection of heavy nuclei. To scan with the simultaneous suppression of the signals from normal (healthy) tissues (e.g., water and fat, the major constituents of the body), the MRI signal from the tissue of interest should be acquired when the longitudinal magnetization of the normal tissues in the "inversion-recovery" mode passes through zero in the course of relaxation, thus providing zero contribution of those tissues to the signal.

We developed algorithms for suppressing signals from up to five different types of tissue that may cover the area of interest (as in the case, for instance, of small neoplasms that are practically invisible against the background of a strong signal from normal tissues). Using these techniques, we were able to obtain effectively filtered MRI images. The image of the semicircular rings and axonal bundle connecting the equilibrium receptors with the neurons of the brain is presented in Fig. 3a 

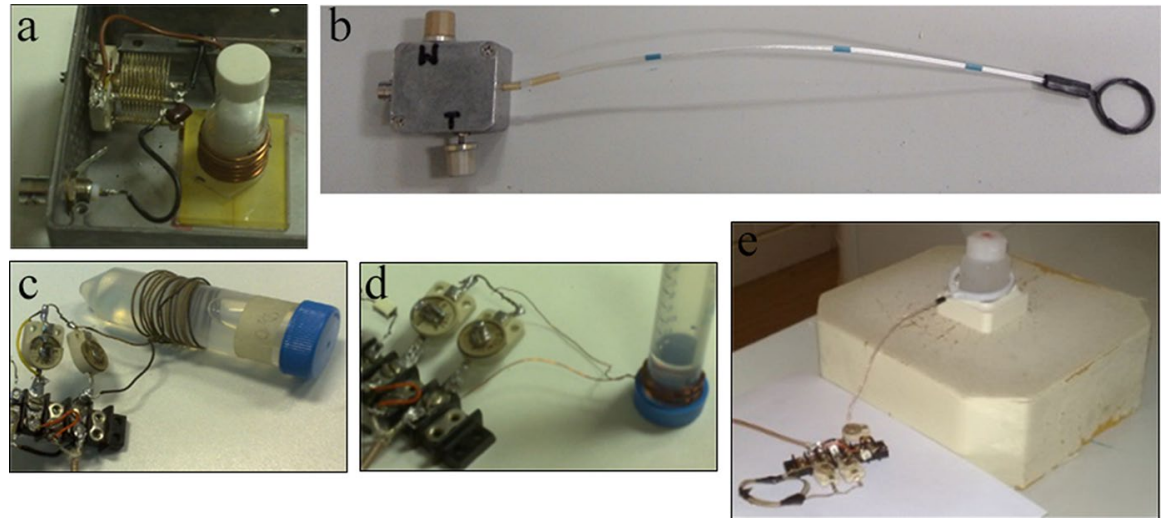

Fig. 2 Radiofrequency (RF) coils designed for multi-nuclear channels of 0.5-T and 7-T scanners [4-7]. The coils operate at the Larmor frequency of the following nuclei: $\mathbf{a}^{35} \mathrm{Cl}$ at $28.1 \mathrm{MHz} ; \mathbf{b}^{2} \mathrm{D}$ at 46.1 MHz; $\mathbf{c}^{2} \mathrm{D}$ at $3.2 \mathrm{MHz} ; \mathbf{d}^{13} \mathrm{C}$ at $5.3 \mathrm{MHz} ; \mathbf{e}^{31} \mathrm{P}$ at $8.5 \mathrm{MHz}$
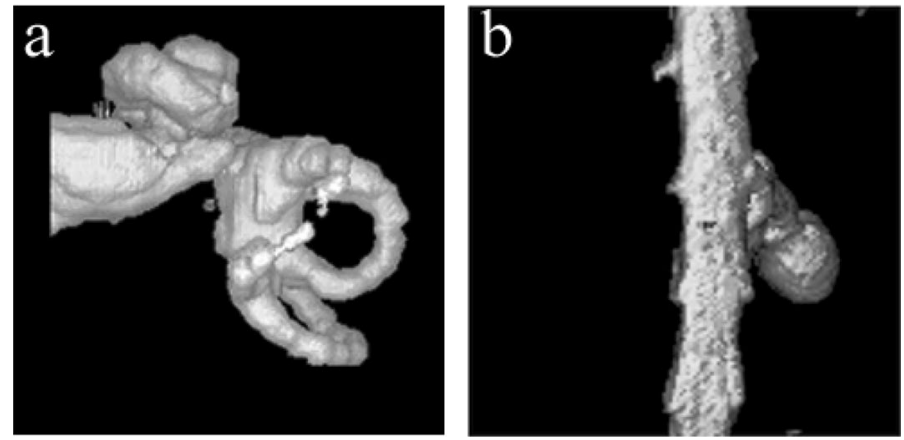

Fig. 3 MRI image of the vestibular apparatus (a) and extracted (virtually) from the spine by suppressing signals from all normal tissues of the spinal cord stem with neurinoma (b) [8]

after being isolated from the image of the whole vestibular system. Figure $3 \mathrm{~b}$ shows the MRI image of a spinal neurinoma obtained after the suppression of the signals from surrounding normal tissues.

Unfortunately, the amplitudes of the MRI signals from heavy nuclei are mostly low, which necessitates the use of special means for increasing them. The most effective method for it is nuclear hyperpolarization [9-11], when nuclear magnetic moments predominantly populate the ground energy level, with the excited states being almost empty. In this case, the noise responses become negligible, and the signal-to-noise ratio (SNR) can be 4-5 orders of magnitude higher than in the regular (at room temperature) state of a magnetized nuclear ensemble. The hyperpolarized state can be created through laser pumping, microwave irradiation of a nuclear ensemble at helium temperatures, and other special methods. For example, filling lungs with hyperpolarized noble gases $\mathrm{He}-3, \mathrm{Xe}-129$ or $\mathrm{Kr}-83$ 
and using MRI at Larmor frequencies of these heavy nuclei allows for clear visualization of lung structures. The researchers from the University of Oxford, UK have recently published the MRI images of the lungs of a healthy patient and of a COVID-19 patient that were filled with hyperpolarized xenon-129 [11]. Unlike widely used computed (X-ray) tomography, in the hyperpolarized MRI images the healthy (bright) areas of the lung tissue and the affected (dark) alveolar areas are clearly separated.

The hyperpolarization method provides a very high quality of images based on the MRI response of heavy nuclei, but at the same time has serious drawbacks. Firstly, the procedure for preparing a hyperpolarized nuclear ensemble is very complicated and requires the use of specialized equipment, with a cost comparable to that of an MRI scanner. Secondly, the required for measurements hyperpolarization state does not last long, for several minutes at best. Therefore, we tasked ourselves with finding such methods of multinuclear MRI diagnostics that would allow, without the use of hyperpolarization, to obtain in limited time clear MRI images of human and animal internal organs and tissues [1-3].

\section{The Most Suitable for This Application Are Substances Containing Fluorine-19 Atoms $\left({ }^{19} \mathrm{~F}\right)$}

An almost complete lack of ${ }^{19} \mathrm{~F}$ in the body tissues allows detecting even the smallest amounts of these substances induced into the body on the negligible background of ${ }^{19} \mathrm{~F}-\mathrm{MRI}$ response from the surrounding tissues. Besides, fluorine-19 nuclei are very similar in their MR properties to protons (Table 1): their Larmor frequency is only $6 \%$ lower than that of proton, and their natural abundance is close to $100 \%$. Thus, after slight frequency adjustment, one can use commercial proton coils of the transmit-receive channel of conventional MRI scanners for ${ }^{19} \mathrm{~F}$ detection (Fig. 4).

Using the approach of detecting MRI signals from fluorine-19 nuclei, we were able to find novel solutions to a number of fundamental and applied biomedical problems [12-18].

Table 1 Parameters of nuclei of the most interest for biomedical research

\begin{tabular}{lllll}
\hline Nucleus & $\begin{array}{l}\text { Gyromagnetic } \\
\text { relation (MHz/T) }\end{array}$ & $\begin{array}{l}\text { Spin quan- } \\
\text { tum number }\end{array}$ & $\begin{array}{l}\text { Natural } \\
\text { content } \\
\text { in } \%\end{array}$ & $\begin{array}{l}\text { Relative } \\
\text { sensitivity }\end{array}$ \\
\hline${ }^{1} \mathrm{H}$ & 42.6 & $1 / 2$ & 99 & 1.0 \\
${ }^{13} \mathrm{C}$ & 10.7 & $1 / 2$ & 1.1 & 0.016 \\
${ }^{17} \mathrm{O}$ & 5.8 & $5 / 2$ & 0.1 & 0.029 \\
${ }^{19} \mathrm{~F}$ & 40.0 & $1 / 2$ & 100 & 0.83 \\
${ }^{23} \mathrm{Na}$ & 11.3 & $3 / 2$ & 100 & 0.093 \\
${ }^{31} \mathrm{P}$ & 17.2 & $1 / 2$ & 100 & 0.07 \\
\hline
\end{tabular}



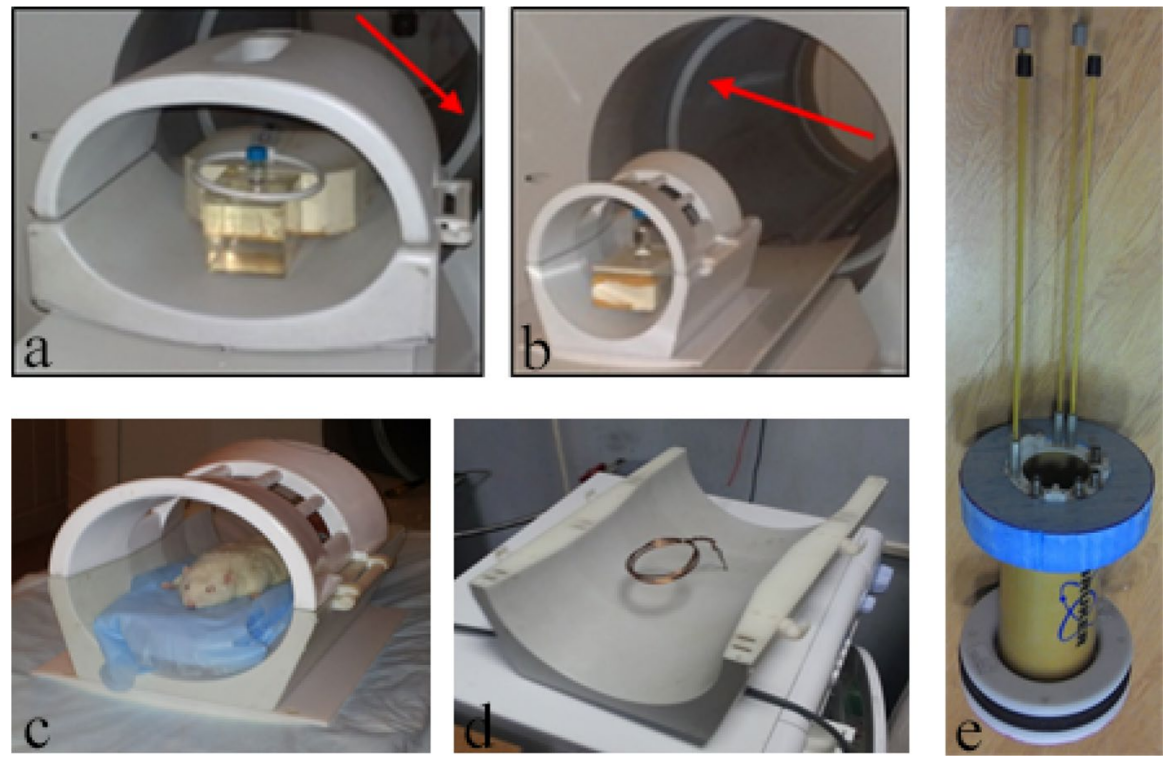

Fig. 4 RF coils for ${ }^{19} \mathrm{~F}$ MRI studies [12-14] operating at $19.83 \mathrm{MHz}$ in $0.5 \mathrm{~T}$ (a-d) and at $283 \mathrm{MHz}$ in $7 \mathrm{~T}(\mathbf{e})$

\subsection{Detecting the Location of Intravenously Injected Blood Substitute Perftoran ${ }^{\circledR}$}

The Perftoran ${ }^{\circledR}$ molecules do not contain protons and thus cannot be imaged with regular clinical MRI scanners. Therefore, prior to our experiments, neither the location of the blood substitute within the body nor its elimination time was known. Experiments on small laboratory animals performed using both the clinical 0.5-T MRI scanner and the 7-T biospectral scanner showed that on the first day after the intraperitoneal or intravenous administration of Perftoran ${ }^{\circledR}$, the drug circulates in the bloodstream, and on the second day it concentrates in the liver and in the spleen (Fig. 5). We also found that Perftoran ${ }^{\circledR}$ is retained in the body for quite a long time, at least a week, and then is excreted naturally, without causing any toxic effects. Since Perftoran ${ }^{\circledR}$ is an even more effective oxygen and carbon dioxide absorber than blood, it can be used for liquid "breathing" by partially filling the lungs with oxygenated Perftoran ${ }^{\circledR}$, a method of artificial respiration alternative to the use of ventilators for patients with severe coronavirus fibrosis of the alveolar system of the lungs.

\subsection{Visualization of the Airways and Lung Structure}

As we already mentioned, the fluorine-19 nucleus is highly sensitive to NMR. Out of all body parts, only bones and teeth enamel contain fluorine naturally, but their solid structure does not allow imaging it by MRI. At the same time, soft 

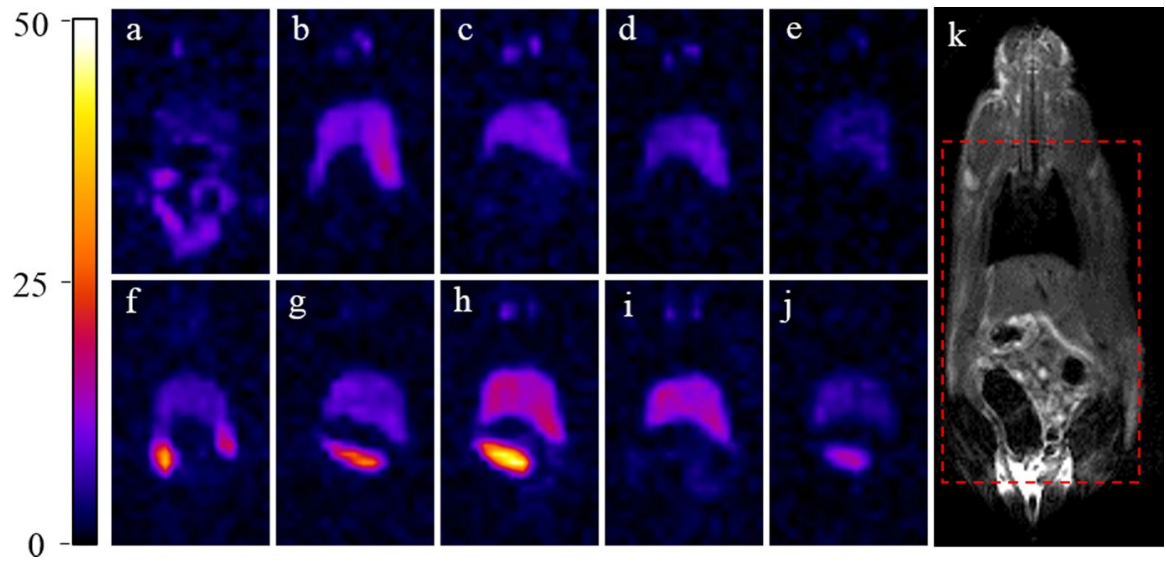

Fig. $5{ }^{19} \mathrm{~F}$ MRI images of rats in $3 \mathrm{~h}(\mathbf{a}, \mathbf{f})$, and then in $2(\mathbf{b}, \mathbf{g}), 3(\mathbf{c}, \mathbf{h}), 6(\mathbf{d}, \mathbf{i})$, and $35(\mathbf{e}, \mathbf{j})$ days after intraperitoneal $(\mathbf{a}-\mathbf{e})$ and intravenous $(\mathbf{f}-\mathbf{j})$ injections of Perftoran ${ }^{\circledR}$; $\mathbf{k}$ the selected ${ }^{1} \mathrm{H}$ MRI slice of a rat. The dashed red box on ${ }^{1} \mathrm{H}$ MRI image corresponds to the field of view for the presented ${ }^{19} \mathrm{~F}$ MRI images. The color scale corresponds to the SNR values in ${ }^{19} \mathrm{~F}$ MRI images. The images were obtained in $7 \mathrm{~T}$ scanner [3-5]

tissues completely lack fluorine and do not produce any MRI signal when scanning is performed at the Larmor frequency of the fluorine-19 nucleus. Thus, the use of various fluorine-containing compounds as contrast agents makes it possible to obtain ${ }^{19} \mathrm{~F}$ MRI images without background and only from those organs or tissues, where the agents (e.g., Perftoran®) accumulate.

An excellent alternative to hyperpolarization methods of lung imaging is the use of fluorine-containing gases as a contrast agent, as they do not require any special preparation before inhaling them. The ${ }^{19} \mathrm{~F}$ MRI diagnostic procedure is inexpensive, easy to perform, and provides good images of the airways. Figure 6 shows ${ }^{19} \mathrm{~F}$ MRI images of the rat lungs obtained on a 7-T scanner [12, 14], and Fig. 7 images of the lungs of a volunteer obtained with a 0.5-T scanner [17].

\section{3 ${ }^{19} \mathrm{~F}$ MRI of a Fluorine Capsule Within the Gastrointestinal Tract (GIT)}

If a patient takes orally a capsule filled with a fluorine-containing drug (perfluorodecalin in our experiments), its 3D location within the GIT can be easily traced with ${ }^{19} \mathrm{~F}$ MRI, with no background signal from the surrounding fluorine-lacking tissues [18] (Fig. 8). Such non-invasive method allows obtaining a three-dimensional image of the GIT structure, studying the motility and patency of the intestines, and, most importantly, performing targeted delivery of drugs packed in the same capsule to the area of intestinal pathology. At the moment when the capsule approaches a neoplasm (say, a cancerous tumor) detected by proton MRI, it is possible, by tracking its passage through the gastrointestinal tract in ${ }^{19} \mathrm{~F}$ MRI mode, to destroy the capsule shell (for example, by focused ultrasound) and release the drug at the tumor site. 

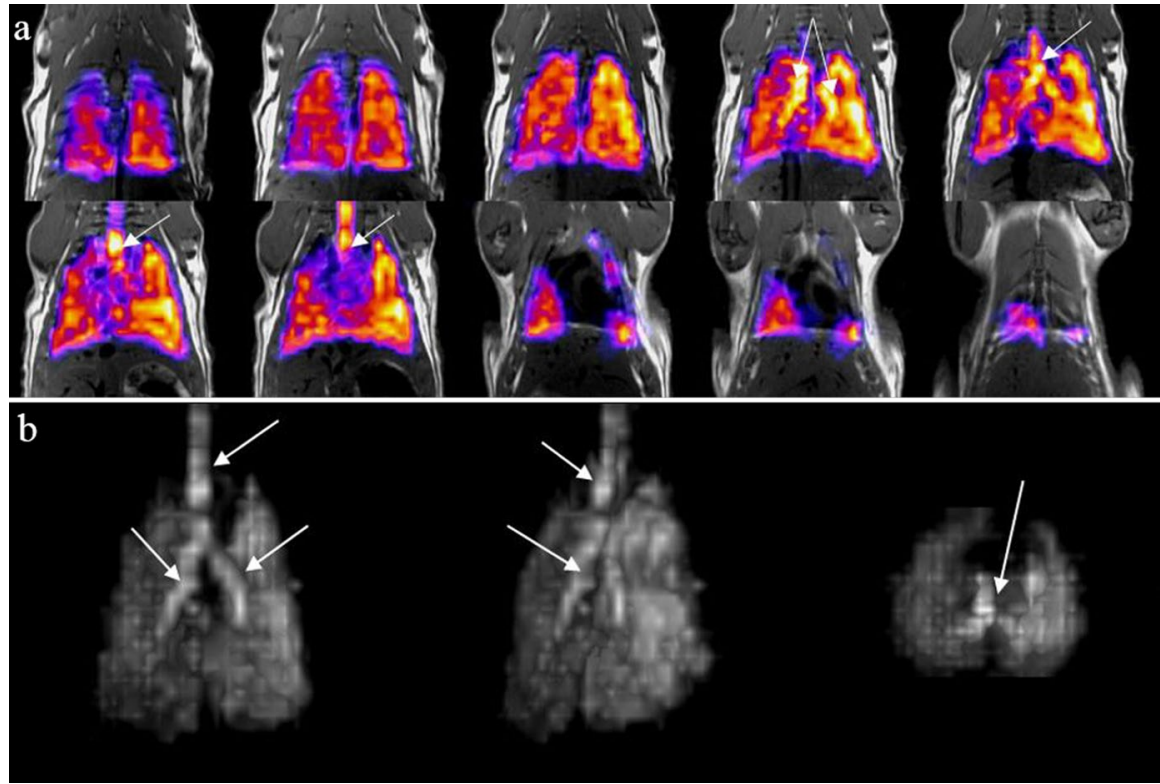

Fig. $6{ }^{19} \mathrm{~F}$ MRI of rat lungs filled with breathing gas mixture of $70 \%$ octafluorocyclobutane C4F8 and $30 \%$ oxygen. a The overlay of ${ }^{19} \mathrm{~F}$ and ${ }^{1} \mathrm{H}$ MRI images in coronal projection; b 3D reconstruction of ${ }^{19} \mathrm{~F}$ MRI images. The arrows point to trachea and bronchia. The images were obtained in 7-T scanner $[12,14]$
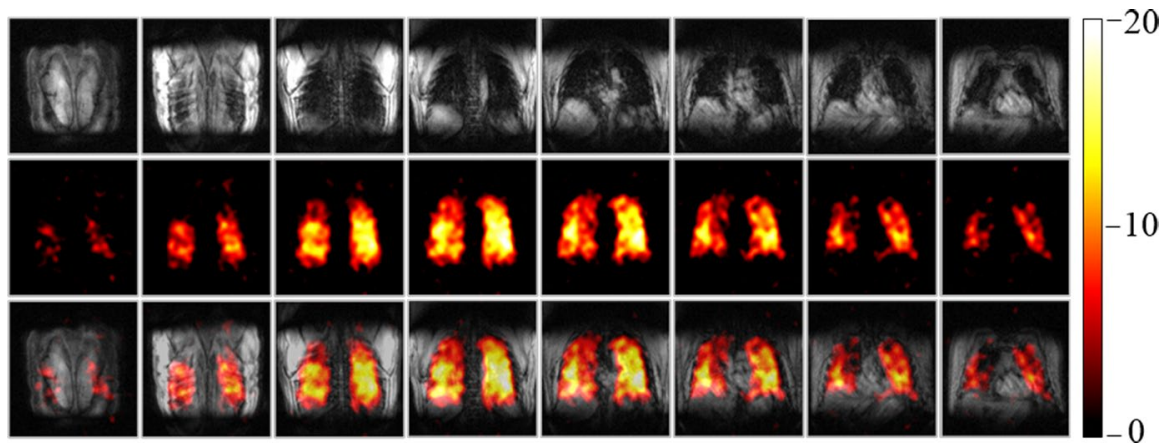

Fig. $7{ }^{19} \mathrm{~F}$ and ${ }^{1} \mathrm{H}$ MRI of human lungs filled with breathing gas mixture of $70 \%$ octafluorocyclobutane and $30 \%$ oxygen. Top row: ${ }^{1} \mathrm{H}$ MRI images; middle row: ${ }^{19} \mathrm{~F}$ MRI images; bottom row: the overlay of ${ }^{1} \mathrm{H}$ and ${ }^{19} \mathrm{~F}$ MRI images. The color scale corresponds to the SNR values in ${ }^{19} \mathrm{~F}$ MRI images. The images were obtained on the medical 0.5-T scanner [17]

Another important task in the multinuclear MRI is detecting the accumulation sites and measuring the concentration of substances containing sodium-23 nuclei [19-21].

Lack or excess of sodium in the body, primarily $\mathrm{NaCl}$ salt, is the cause of widespread socially significant diseases-blood pressure disorders (hyper- or hypotension), diabetes, renal failure, cholelithiasis, etc. With the help of an MRI scanner 

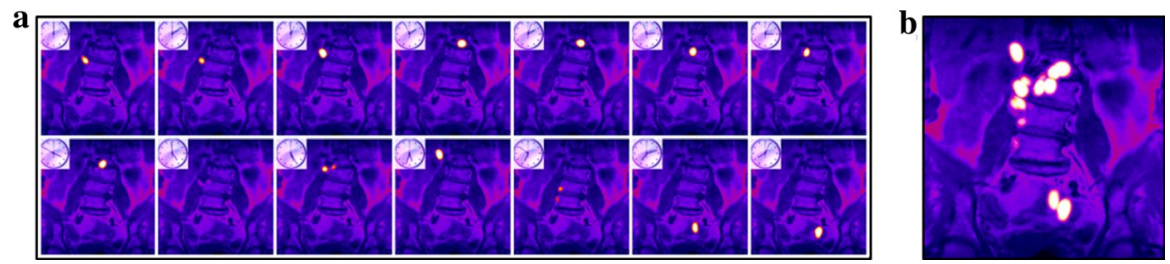

Fig. 8 a The overlay of ${ }^{19} \mathrm{~F}$ and ${ }^{1} \mathrm{H}$ MRI images of a volunteer abdomen obtained at different time points (from 0 to $8 \mathrm{~h}$ ) after oral administration of a capsule with perfluorotributylamine. b The overlay of the presented separate images. The images were obtained in $0.5 \mathrm{~T}$ [18]
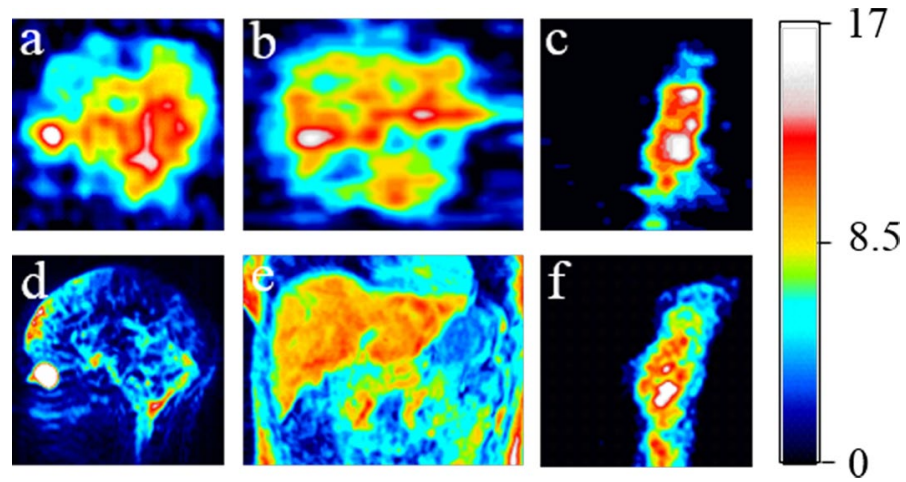

Fig. $9{ }^{23} \mathrm{Na}$ (top row) and ${ }^{1} \mathrm{H}$ (bottom row) MRI of human organs-head (a, d), liver (b, e) and foot (c, f). The color scale corresponds to the SNR values in MRI images [21]

tuned to the resonance frequency of sodium-23 nuclei, it is possible to non-invasively measure sodium concentration in various parts of the human body, as shown, for example, in Fig. 9.

\section{Conclusion}

The presented examples clearly demonstrate that multinuclear MRI has a great potential in fundamental research and in clinical fields. The biomedical value of many heavy nuclei that are part of drugs, contrast agents, and molecular complexes in live tissues, emphasizes the need for creating and using MRI scanners tuned to the Larmor frequencies of such nuclei. The data obtained and partially presented in this review were achieved jointly with our colleagues-employees and students of some faculties of the Lomonosov Moscow State University (physical, chemical, fundamental medicine, biological, etc.) on the equipment of the Center for Magnetic Tomography and Spectroscopy. The main characters and co-authors of these multinuclear MRI studies are Nikolay Anisimov, Dr.Sci. (Phys. and Math.), Senior Researcher at the Faculty of Fundamental Medicine (FFM), MSU; Mikhail Gulyaev, PhD (Phys. and Math.), Senior Researcher at the FFM, MSU; Olga Pavlova, PhD (Phys. and Math.), Researcher at the Faculty of Physics and at the FFM, MSU; 
Dmitry Volkov, PhD student of the Faculty of Physics, MSU; Elnur Sadykhov, $\mathrm{PhD}$ student of the Moscow Engineering Physics Institute; Lev Gervits, Senior Researcher at the Nesmeyanov Elemento-Organic Compound Institute of Russian Academy of Sciences. It should also be noted that the initiator of the sodium MRI studies carried out at the CMTS was the co-author of some of our publications, Associate Professor of the University of Nottingham (Great Britain), PhD (Phys. and Math.) Galina Pavlovskaya, coordinator of the agreement on joint scientific and educational activity between the Moscow State University and the University of Nottingham.

Funding The presented research was supported by the Russian Foundation for Basic Research (Grants 19-29-10015 and 20-52-10004), as well as by the Interdisciplinary Scientific and Educational School of Moscow University "Photonic and Quantum Technologies. Digital Medicine".

\section{References}

1. N.V. Anisimov, M.V. Gulyaev, O.S. Pavlova, D.V. Volkov, L.L. Gervits, Yu.A. Pirogov, Multinuclear magnetic resonance imaging in biomedical experiments. J. Phys. Conf. Ser. 886, 012006 (2017)

2. N.V. Anisimov, O.S. Pavlova, A.G. Agafonnikova, A.V. Kosenkov, D.V. Fomina, Multinuclear applications on 0.5T magnetic resonance scanner. Appl. Magn. Reson. 50(1-3), 17-27 (2019)

3. Yu.A. Pirogov, Multinuclear magnetic resonance imaging and NMR spectroscopy in biomedical investigations. Phys. Procedia. 82, 3-7 (2016)

4. N.V. Anisimov, O.S. Pavlova, D.V. Volkov, M.V. Gulyaev, Yu.A. Pirogov, Registration of nuclei other than protons on a $0.5 \mathrm{~T}$ magnetic resonance scanner. J. Radioelectron. (Electron. J.). 11, 1-9 (2015). (in Russian)

5. N.V. Anisimov, M.V. Gulyaev, D.V. Volkov, O.S. Pavlova, Y.A. Pirogov, Studies of animals by magnetic resonance imaging. multinuclear aspects. Izvestiya Vuzov Physics. 58(12), 5-10 (2015)

6. A.V. Protopopov, M.V. Gulyaev, O.S. Pavlova, E.A. Mokhova, Yu.A. Pirogov, Computation of the resonance frequencies of the transmission line resonators used in MRI. Magn. Reson. Imaging. 61, 167-174 (2019)

7. M.V. Gulyaev, O.S. Pavlova, D.V. Volkov, E.G. Sadykhov, N.V. Anisimov, Y.A. Pirogov, Application of copper plates for frequency tuning of surface wired and wireless MRI coils. J. Magn. Reson. 309, 106626 (2019)

8. N.V. Anisimov, S.S. Batova, Y.A. Pirogov, Magnetic Resonance Imaging: Contrast Management and Interdisciplinary Applications (MAKS Press, Moscow, 2013), p. 244. (in Russian)

9. A.-M. Oros, N.J. Shah, Hyperpolarized xenon in NMR and MRI // Phys. Med. Biol. IOP Publishing 49(20), R105 (2004)

10. S.B. Fain, F.R. Korosec, J.H. Holmes, R. O’Halloran, R.L. Sorkness, T.M. Grist, Functional lung imaging using hyperpolarized gas MRI. J. Magn. Reson. Imaging. 25(5), 910-923 (2007)

11. https://www.bbc.com/news/health-55017301 Oxford Univ. Accessed 1 Dec 2020

12. O.S. Pavlova, V.N. Semenova, M.V. Gulyaev, L.L. Gervits, Y.A. Pirogov, Visualization of the respiratory system of laboratory animals by MRI on fluorine nuclei. J. Radio Electron. 11, 1-11 (2018). (in Russian)

13. N.V. Anisimov, M.V. Gulyaev, D.V. Volkov, S.S. Batova, O.S. Pavlova, D.V. Fomina, Y.A. Pirogov, Optimization of MRI parameters for the gradient echo method in fluorocarbon research. Phys. Wave Phenom. 25(2), 140-146 (2017)

14. O.S. Pavlova, D.V. Volkov, M.V. Gulyaev, M.S. Kostromina, L.L. Gervits, N.V. Anisimov, Y.A. Pirogov, Magnetic resonance imaging of the lungs on fluorine-19 nuclei using perfluorocyclobutane gas. Med. Phys. 75(4), 59-64 (2017). (in Russian)

15. M.V. Gulyaev, A.V. Kuznetsova, D.V. Silachev, T.I. Danilina, L.L. Gevits, Y.A. Pirogov, Realization of 19F MRI oximetry method. Magn. Reson. Mater. Phys. 32, 307-315 (2019) 
16. O.S. Pavlova, M.V. Gulyaev, N.V. Anisimov, D.N. Silachev, L.L. Gervits, Y.A. Pirogov, New aspects of biodistribution of perfluorocarbon emulsions in rats: thymus imaging. Appl. Magn. Reson. 51, $1625-1635$ (2020)

17. O.S. Pavlova, N.V. Anisimov, L.L. Gervits, M.V. Gulyaev, V.N. Semenova, Y.A. Pirogov, V.Y. Panchenko, 19F MRI of human lungs at 0.5 Tesla using octafluorocyclobutane. Magn. Res. Med. 84, 2117-2123 (2020)

18. D.V. Volkov, N.V. Anisimov, L.L. Gervits, M.V. Gulyaev, Y.A. Pirogov, Study of the gastrointestinal tract of humans and animals using the 19F MRI method. Med. Phys. 75(3), 62-68 (2017). (in Russian)

19. N.V. Anisimov, E.G. Sadykhov, O.S. Pavlova, D.V. Fomina, Y.A. Pirogov, Whole body sodium 0.5 T MRI using surface coil and long time echo. Appl. Magn. Reson. 50(10), 1149-1161 (2019)

20. N.V. Anisimov, A.A. Tarasova, O.S. Pavlova, D.V. Fomina, A.M. Makurenkov, G.E. Pavlovskaya, Y.A. Pirogov, MRI coils optimized for detection of $1 \mathrm{H}$ and $23 \mathrm{Na}$ at $0.5 \mathrm{~T}$. Appl. Magn. Res. 52, $1-13(2021)$

21. N.V. Anisimov, A.A. Tarasova, Y.A. Pirogov, Receiving and transmitting coils for recording sodium signals on a $0.5 \mathrm{~T}$ magnetic resonance imager. Electromagn. Waves Electron. Syst. 25(4), 28-36 (2020)

Publisher's Note Springer Nature remains neutral with regard to jurisdictional claims in published maps and institutional affiliations. 